\title{
Comparison of a video versus paper questionnaire on functional limitation in lower limb osteoarthritis
}

L. Dubouis ${ }^{1}$, W. Ngueyon-Sime1 ${ }^{1}$, W. Peter ${ }^{2}$, A. Vallata ${ }^{1}$, J. Epstein ${ }^{1,3}$, A-C. Rat ${ }^{1,3}$, N. Agrinier ${ }^{1,3}$, C. B. Terwee ${ }^{2}$ and F. Guillemin ${ }^{1,3^{*}}$

\begin{abstract}
Introduction: The video Animated Activity Questionnaire (AAQ) was developed to assess the impact of lower limb osteoarthritis (OA) on daily functional activities. The objective of the study was to compare the video and the HOOS/KOOS paper questionnaires and to assess the effect of order of administration.

Material and methods: Patients recruited in the KHOALA cohort were randomized in two groups: AAQ questionnaire first (AAQ-first group) and HOOS (hip)/KOOS (knee) questionnaire first (H/KOOS-first group). Within group differences between $\mathrm{AAQ}$ and $\mathrm{HOOS} / \mathrm{KOOS}$ scores were compared using a Student t-test. The Spearman correlation coefficient between AAQ score and $\mathrm{HOOS} / \mathrm{KOOS}$ score was calculated in each group then compared, using Fisher z-transformation.

Results: Among 200 randomized patients, 188 (65.8 years, 66.0\% women) completed the questionnaires: 99 in the AAQfirst group and 89 in the $\mathrm{H} / \mathrm{KOOS}$-first group. The AAQ score was 85.9 (SD: 13.7) in the AAQ-first versus 87.8 (SD: 13.1) in the H/KOOS-first group ( $p=0.34$ ). The H/KOOS score was 72.5 (SD: 21.2) in the AAQ-first versus 73.5 (SD: 18.4) in the H/KOOSfirst group $(p=0.71)$. The Spearman correlation coefficient between AAQ and H/KOOS in the AAQ-first was 0.84[0.77-0.89] and $0.73[0.61-0.81]$ in $\mathrm{H} / \mathrm{KOOS}$-first group. These correlations differed between groups significantly $(p=0.02)$.

Conclusion: This study found video AAQ and paper HOOS/KOOS questionnaire highly correlated, with a moderate but significant effect of order administration of video and paper questionnaires evidencing a stronger correlation when the videos were viewed first.
\end{abstract}

Keywords: Questionnaire, Osteoarthritis, Function, Video

\section{Introduction}

Osteoarthritis (OA) is the most frequent joint disease. The hip and knee OA, the most disabling, account for 9 , $1 \%$ of women (about 3 million women) and $6,6 \%$ of men (about 2 million men) among 40-75 years old in France [1]. OA progresses by flare followed by chronic pain with an impact on daily activity and function. Several methods are available to assess this impact. For instance, performance-based tests [2, 3], where a health professional observes OA impact on patients daily activities. They are difficult to conduct on a regular basis, requiring

\footnotetext{
* Correspondence: francis.guillemin@univ-lorraine.fr

${ }^{1}$ CHRU-Nancy, Inserm, Université de Lorraine CIC Clinical epidemiology, Nancy, France

${ }^{3}$ Université de Lorraine, APEMAC, Nancy, France

Full list of author information is available at the end of the article
}

staff personal, and are costly. Patient Reported Outcome Measures (PROMs) [4] have been developed to allow patient self-evaluation. In OA the Hip disability Osteoarthritis Outcome Score (HOOS) [5] and the Knee injury Osteoarthritis Score (KOOS) [6] are commonly used. The degree of severity expressed is then depending on the representation the patient has of her/his disease [7].

In order to correctly assess the impact of OA on daily basic functional activities such as walking, rising and sitting down, and stair climbing, an interactive questionnaire has been developed in close collaboration with two focus group of hip and knee OA patients [8]. The Animated Activity Questionnaire (AAQ) $[8,9]$ is a computerized video questionnaire that assesses daily basic activity limitations. The psychometric properties of the AAQ have been established in an international study $[8,9]$ demonstrating its

(c) The Author(s). 2019 Open Access This article is distributed under the terms of the Creative Commons Attribution 4.0 International License (http://creativecommons.org/licenses/by/4.0/), which permits unrestricted use, distribution, and reproduction in any medium, provided you give appropriate credit to the original author(s) and the source, provide a link to the Creative Commons license, and indicate if changes were made. The Creative Commons Public Domain Dedication waiver (http://creativecommons.org/publicdomain/zero/1.0/) applies to the data made available in this article, unless otherwise stated. 
reproducibility and expected correlations between paper and video questionnaires, administered in this order, and scale calibration thanks to differential item functioning (DIF) analysis [10].

One may hypothesize that the video questionnaire, when administered first, influences the responses of the patients to paper questions on their abilities and functional capacities. Randomizing the order of administration will allow detecting such an effect and, in contrast, asserting independent measurement of each questionnaire if none is evidenced.

The objective of this study was to assess the effect of order of administration of video AAQ and paper HOOS/KOOS questionnaires on responses.

\section{Patients and methods Sampling}

This study was an ancillary analysis of an international validation study of the AAQ questionnaire [10] in which we introduced a randomization of order of administration in the French sample, conducted from March 2014 to June 2015.

Patients were recruited in the KHOALA cohort [11], aged 40 to 75 years at inclusion in the cohort, and presenting knee or hip OA diagnosed and confirmed by a rheumatologist according to American College of Rheumatology (ACR) Clinical Criteria [12, 13]. Exclusion criteria were: unable to fill in questionnaires, no informed consent. Furthermore to be included in the present study, they had to receive some treatment for osteoarthritis in the past 12 months.

The sample size was imposed on by the international multi-country protocol. Among 200 randomized patients, 189 patients were eligible to complete the questionnaires in the 2 groups ( 90 in HOOS/KOOS-first and 99 in AAQ-first groups). One patient from the HOOS/ KOOS-first group with missing data was excluded.

\section{Ethical approval}

This study was approved by the Medical Ethics Committee of France (CPP Est III Nancy) and institutional national review board (CNIL). The study complied with the Declaration of Helsinki. Patients provided written informed consent.

\section{Randomization}

Patients satisfying inclusion criteria were computer randomized at the Inserm CIC coordinating center in two arms; AAQ questionnaire first followed by $\mathrm{H} / \mathrm{KOOS}$ questionnaire (AAQ-first group) or $\mathrm{H} / \mathrm{KOOS}$ questionnaire first followed by AAQ questionnaire (H/KOOSfirst group).

\section{Data}

Data were collected before a clinic visit, alone in the room. They included socio-economic characteristics: age, sex, BMI; clinical characteristics: symptomatic joint (hip, knee or both), side (right, left or both) and pain (VAS); and PROMs questionnaires without missing data (HOOS, KOOS, and AAQ).

The score of HOOS and KOOS questionnaires, referring to the more symptomatic joint, contains 17 items each scored 0 to 4 . The full score obtained by the sum of items was standardized to $0-100$ points scale with higher score representing less limitations in daily activity.

The AAQ video questionnaire is composed of 17 videos showing daily life situations: going up and downstairs (2 items); walking outside on a flat surface (1 item); walking outside on uneven terrain (1 item); walking inside (1 item); walking up or down a slope (2 items); picking up an object from floor (1 item); rising and sitting down on the floor (1 item); rising and sitting on the chair, sofa, and toilet (6 items); putting on and taking off shoes (2 items). For each activity, three to five videos show to patient the levels of difficulty. In addition a response option 'unable to perform the activity' is given. For each activity all videos are shown on the same screen to facilitate comparison from the first with optimal performance to the last with the maximum level of disability. Videos are visualized as many times as needed. The patient must chose the video that best corresponds to how he/she is performing that activity. A research nurse was available for guidance and checked patients filled in the questionnaire until the end item.

Each activity is scored from 0 to 4,5 or 6 according to the number of videos, and the option 'unable to perform'. Each activity score is translated on a 0 to 100 scale. The total AAQ score is the sum of all activities divided by 17 . The higher score on the 0 to 100 scale corresponds to less activity limitations.

The HOOS, KOOS and AAQ questionnaires have shown good validity, internal consistency, test-retest reliability in source and French languages [5, 6, 8-10].

\section{Statistics}

Patients baseline characteristics were described per group. The characteristics of these 2 groups were compared using the Chi-square test for qualitative variables. The quantitative variables, and the within group difference between the AAQ and HOS/KOOS scores were compared Student $t$ test.

The Spearman correlation coefficient between AAQ score and HOOS/KOOS score was calculated in each of the 2 groups to assess the intra-group correlation. These coefficients were then compared after Fisher z-transformation by a Student $t$ test. 
Since the sample size was constrained by the international study protocol, a post-hoc power was calculated.

Statistical analysis was done using SAS Version 9.3, Cary NC, USA.

\section{Results}

A total of 188 patients with complete data were included (Fig. 1). Mean age was 65.8 years with 2:1 women to men ratio. The patients who answered the questionnaire were significantly older than those who did not ( 65.8 vs 58.9 years; $p<0.0001$ ) and showed no difference in sex, level of education and BMI. The mean AAQ score was 86.8 (SD: 13.4). The mean HOOS/KOOS was 73.0 (SD: 19.9) and the mean difference between AAQ and HOOS/KOOS was 13.8 (SD: 11.9).

Patients characteristics in each randomized group showed no difference (Table 1). Neither the AAQ scores in the AAQ-first group versus in the HOOS/KOOS-first group $(p=0.34))$, nor the HOOS/KOOS scores in the AAQ-first group versus in the HOOS/KOOS-first group $(p=0.71))$ differed.

The Spearman correlation coefficient between AAQ and HOOS/KOOS questionnaires was $0.84[0.77-0.89]$ in the AAQ-first and 0.73 [0.61-0.81] in the $\mathrm{H} / \mathrm{KOOS}$-first group and these coefficients differed significantly $(p=$ 0.02). These results did not differ by hip or knee joint (data not shown). The power of this analysis was $93.3 \%$.

\section{Discussion}

This study found a significant effect of order administration of video AAQ and paper HOOS/KOOS questionnaires. The AAQ and HOOS/KOOS scores were more
Table 1 Comparison of groups according to questionnaires order of administration $(n=188)$

\begin{tabular}{|c|c|c|c|c|c|c|c|}
\hline & \multirow{2}{*}{\multicolumn{3}{|c|}{$\frac{\text { AAQ-first group }}{N=99(52.7 \%)}$}} & \multirow{2}{*}{\multicolumn{3}{|c|}{$\begin{array}{l}\text { HOOS/KOOS-first group } \\
N=89(47.3 \%)\end{array}$}} & \multirow[b]{3}{*}{$p^{b}$} \\
\hline & & & & & & & \\
\hline & $\bar{N}$ & $\% /$ mean & $\overline{S D^{a}}$ & $\bar{N}$ & $\% /$ mean & $S D^{a}$ & \\
\hline Age & 99 & 65.9 & 7.5 & 89 & 65.6 & 6.9 & 0.75 \\
\hline Sex & & & & & & & 0.69 \\
\hline Male & 35 & 35.4 & & 29 & 32.6 & & \\
\hline Female & 64 & 64.6 & & 60 & 67.4 & & \\
\hline Body Mass Index & 97 & 28.4 & 5.3 & 88 & 28.6 & 6.6 & 0.82 \\
\hline Level of education & & & & & & & 0.39 \\
\hline Primary & 12 & 12.1 & & 17 & 19.1 & & \\
\hline Secondary & 51 & 51.5 & & 40 & 44.9 & & \\
\hline Superior & 36 & 36.4 & & 32 & 36.0 & & \\
\hline Pain & 99 & 3.5 & 2.3 & 89 & 3.4 & 2.3 & 0.86 \\
\hline Site & & & & & & & 0.56 \\
\hline Hip & 28 & 28.3 & & 21 & 23.6 & & \\
\hline Knee & 60 & 60.6 & & 54 & 60.7 & & \\
\hline Both & 11 & 11.1 & & 14 & 15.7 & & \\
\hline Side & & & & & & & 0.95 \\
\hline Right & 39 & 39.4 & & 35 & 39.3 & & \\
\hline Left & 36 & 36.4 & & 34 & 38.2 & & \\
\hline Both & 24 & 24.2 & & 20 & 22.5 & & \\
\hline AAQ score & 99 & 85.9 & 13.7 & 89 & 87.8 & 13.1 & 0.34 \\
\hline HOOS/KOOS score & 99 & 72.5 & 21.2 & 89 & 73.5 & 18.4 & 0.71 \\
\hline
\end{tabular}

${ }^{a} S D$ Standard deviation

${ }^{\mathrm{b}}$ Chi-Square test for qualitative variables and Student $\mathrm{t}$ test for quantitative variables

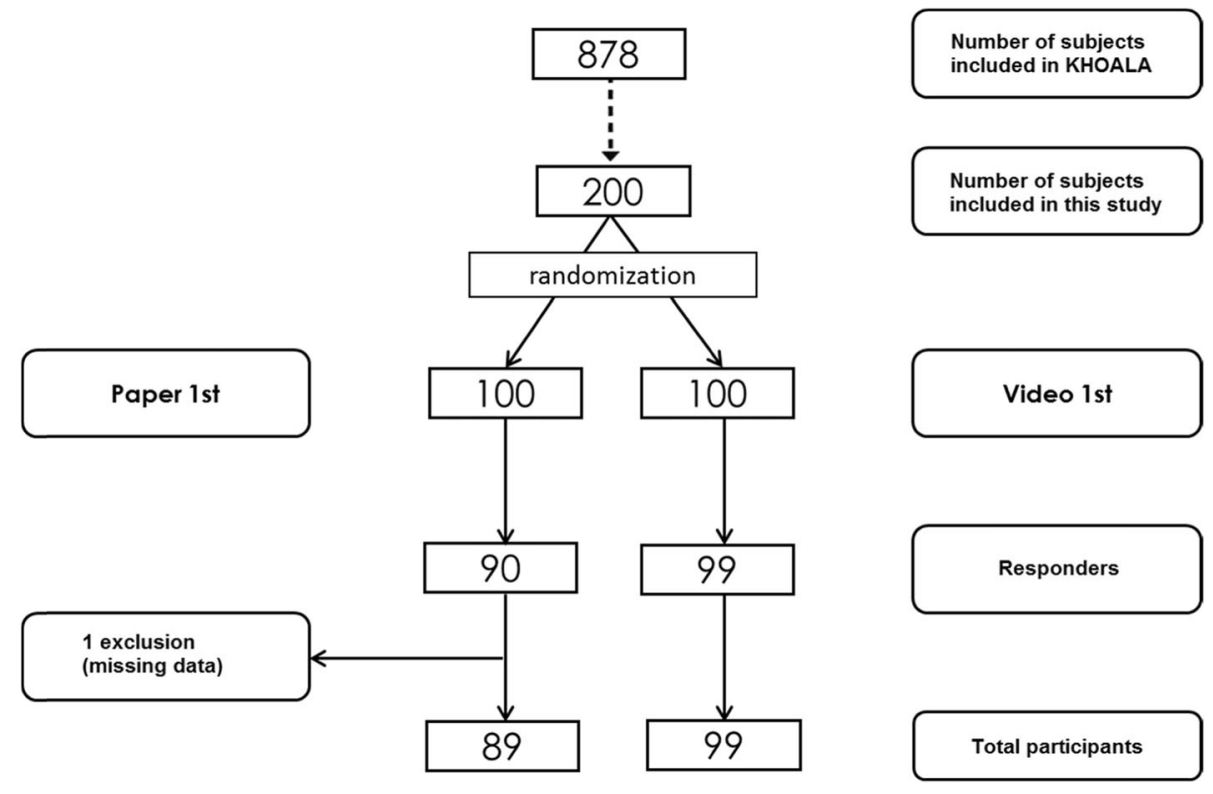

Fig. 1 Patient's selection from the KHOALA cohort 
highly correlated in patients who completed the AAQ first, indicating that seeing the video images first may have a stronger impact on answering paper questionnaire than the reverse in the domain of physical mobility. The absence of difference in correlations would have suggested that the two questionnaires, measuring different though correlated constructs, influence each other equally.

To be consistent, patients may adapt their responses to the second questionnaire depending on responses given to the first, i.e. a halo effect [14], modifying their perception to evaluate specific aspects of functional limitation contained in the second questionnaire. When a patient completes several questionnaires (with the same answer scale but on a different administration mode), the subject tends unconsciously to modify his answers to the second questionnaire so that they correspond to the first and thus be faithful to itself. Moreover, it is likely that showing the video representation first imprints responses to the paper second while reciprocal is less true assuming that videos has more impact than words [15].

In this experiment, the absolute difference in scores between the two questionnaires cannot be interpreted, since the exact correspondence in content between the two constructs is not the same, and neither are the metrics used.

The AAQ questionnaire combines advantages of PROMs and of performance-based tests without most of their inconveniences: decrease of inter-subjects variability for interpretation, independence of patient motivation to do the test, videos referring to activities in the past week and not to a single time of the test performance. The cost is low and the comprehension is easier as it is not text dependent. However, its impact on the responses to subsequent questionnaires should be kept in mind.

This study has some limitations. Although the patients were sampled in a national cohort representative of French knee and hip osteoarthritis, it was only one country and the results may not be fully extrapolated to other. However, the international AAQ validity study [9] has shown the measurement robustness of the AAQ instrument. Therefore, it is plausible that these results can be generalized. Second, the absence of a gold standard (like an external observer quoting the patients performances) does not allow us to infer whether one measure is less biased than the other. Another strengths of this study is its statistical power (93.3\%).

\section{Conclusion}

To conclude, the AAQ and HOOS/KOOS scores were more highly correlated in patients who completed the AAQ first than in patients who completed the HOOS/ KOOS first, indicating a stronger association when videos are viewed first.

\section{Acknowledgements}

Not applicable.

\section{Authors' contributions}

LD interpreted the data and drafted the first version of the manuscript. WNS run the statistical analyses and interpreted the data. CBT revised the statistical plan and interpreted the data. ACR and WP contributed to the design of the study and interpreted the data. NA, JE interpreted the data. AV provided technical support. FG designed and supervised the study, and interpreted the data. All authors contributed in the critical revision of the article for important intellectual content and all authors approved the final manuscript.

\section{Funding}

This work was supported by European League Against Rheumatism (EULAR). EULAR had neither role in the design of the study, collection, analysis, and interpretation of data nor in writing the manuscript.

\section{Availability of data and materials}

The datasets used and analysed during the current study are available from the corresponding author on reasonable request.

\section{Ethics approval and consent to participate}

This study was approved by the Medical Ethics Committee of France (CPP Est III Nancy) and institutional national review board (CNIL). The study complied with the Declaration of Helsinki. Patients provided written informed consent.

\section{Consent for publication}

Not applicable.

\section{Competing interests}

The authors declare that they have no competing interests.

\section{Author details}

${ }^{1} \mathrm{CHRU}-$ Nancy, Inserm, Université de Lorraine CIC Clinical epidemiology, Nancy, France. ${ }^{2}$ Amsterdam UMC, Vrije Universiteit Amsterdam, Department of Epidemiology and Biostatistics, Amsterdam Public Health Research Institute, de Boelelaan 1117, Amsterdam, the Netherlands. ${ }^{3}$ Université de Lorraine, APEMAC, Nancy, France.

Received: 18 January 2019 Accepted: 9 October 2019

Published online: 03 November 2019

\section{References}

1. Guillemin F, Rat AC, Mazieres B, Pouchot J, Fautrel B, Euller-Ziegler L, et al. Prevalence of symptomatic hip and knee osteoarthritis: a two-phase population-based survey1. Osteoarthr Cartil. 2011;19(11):1314-22.

2. Dobson F, Hinman RS, Roos EM, Abbott JH, Stratford P, Davis AM, et al. OARSI recommended performance-based tests to assess physical function in people diagnosed with hip or knee osteoarthritis. Osteoarthr Cartil. 2013; 21(8):1042-52.

3. Bennell K, Dobson F, Hinman R. Measures of physical performance assessments: self-paced walk test (SPWT), stair climb test (SCT), six-minute walk test (6MWT), chair stand test (CST), timed up \& go (TUG), sock test, lift and carry test (LCT), and Car task. Arthritis Care Res. 2011;63(S11):S350-70.

4. Maly MR, Costigan PA, Olney SJ. Determinants of self-report outcome measures in people with knee osteoarthritis. Arch Phys Med Rehabil. 2006; 87(1):96-104.

5. Ornetti P, Parratte S, Gossec L, Tavernier C, Argenson J-N, Roos EM, et al. Cross-cultural adaptation and validation of the French version of the hip disability and osteoarthritis outcome score (HOOS) in hip osteoarthritis patients. Osteoarthr Cartil. 2010;18(4):522-9.

6. Ornetti P, Parratte S, Gossec L, Tavernier C, Argenson J-N, Roos EM, et al. Cross-cultural adaptation and validation of the French version of the knee injury and osteoarthritis outcome score (KOOS) in knee osteoarthritis patients. Osteoarthr Cartil. 2008;16(4):423-8.

7. Büchi S, Sensky T, Sharpe L, Timberlake N. Graphic representation of illness: a novel method of measuring patients' perceptions of the impact of illness. Psychother Psychosom. 1998;67(4-5):222-5. 
8. Peter WF, Loos M, van den Hoek J, Terwee CB. Validation of the animated activity questionnaire (AAQ) for patients with hip and knee osteoarthritis: comparison to home-recorded videos. Rheumatol Int. 2015;35(8):1399-408.

9. Peter WF, Loos M, de Vet HCW, Boers M, Harlaar J, Roorda LD, et al.

Development and preliminary testing of a computerized animated activity questionnaire in patients with hip and knee osteoarthritis. Arthritis Care Res. 2015;67(1):32-9.

10. Peter WF, de Vet HC, Boers M, Harlaar J, Roorda LD, Poolman RW, Scholtes VA, Steultjens M, Hendry GJ, Roos EM, Guillemin F, Benedetti MG, Cavazzuti L, Escobar A, Dagfinrud H, Terwee CB. Cross-Cultural and Construct Validity of the Animated Activity Questionnaire. Arthritis Care \& Research. 2017;69: 1349-59. https://doi.org/10.1002/acr.23127.

11. Guillemin F, Rat A-C, Roux CH, Fautrel B, Mazieres B, Chevalier X, et al. The KHOALA cohort of knee and hip osteoarthritis in France. Joint Bone Spine. 2012;79(6):597-603.

12. Altman R, Alarcón G, Appelrouth D, Bloch D, Borenstein D, Brandt K, et al. The American College of Rheumatology criteria for the classification and reporting of osteoarthritis of the hip. Arthritis Rheum. 1991;34(5):505-14.

13. Altman R, Asch E, Bloch D, Bole G, Borenstein D, Brandt K, et al. Development of criteria for the classification and reporting of osteoarthritis: classification of osteoarthritis of the knee. Arthritis Rheum. 1986;29(8):1039-49.

14. Streiner DL, Norman GR. Health Measurement Scales: A practical guide to their development and use. 5th ed. Oxford: Oxford University Press; 2008. p. 394.

15. Alain L. Mémoire des images et double codage. L'année psychologique. 1995;95(4):661-73.

\section{Publisher's Note}

Springer Nature remains neutral with regard to jurisdictional claims in published maps and institutional affiliations.

Ready to submit your research? Choose BMC and benefit from:

- fast, convenient online submission

- thorough peer review by experienced researchers in your field

- rapid publication on acceptance

- support for research data, including large and complex data types

- gold Open Access which fosters wider collaboration and increased citations

- maximum visibility for your research: over $100 \mathrm{M}$ website views per year

At $\mathrm{BMC}$, research is always in progress.

Learn more biomedcentral.com/submissions 